\title{
Dry Eye Disease in Hemifacial Spasm Patients Treated with Botulinum Toxin Type A
}

\author{
Supharat Jariyakosol (iD ${ }^{1,2}$ \\ Lita Uthaithammarat ${ }^{1,2}$ \\ Nattakarn Chatwichaikul ${ }^{1,2}$ \\ Ngamjit Kasetsuwan ${ }^{1-3}$ \\ Yuda Chongpison iD ${ }^{4}$ \\ 'Department of Ophthalmology, Faculty \\ of Medicine, Chulalongkorn University, \\ Bangkok, Thailand; ${ }^{2}$ Ophthalmology \\ Department, King Chulalongkorn \\ Memorial Hospital, Thai Red Cross \\ Society, Bangkok, Thailand; ${ }^{3}$ Center of \\ Excellence for Cornea and Stem Cell \\ Transplantation, Department of \\ Ophthalmology, Faculty of Medicine, \\ Chulalongkorn University, Bangkok, \\ Thailand; ${ }^{4}$ Biostatistic Excellence Center, \\ Research Affairs, Faculty of Medicine, \\ Chulalongkorn University, Bangkok, \\ Thailand
}

Purpose: To assess the impact of botulinum toxin type A (BTX-A) on signs and symptoms of dry eye (DE) in affected eye of hemifacial spasm (HFS) patients and to compare the prevalence of DE between affected and non-affected eye in HFS patients.

Patients and Methods: This prospective study included participants with unilateral HFS, who received BTX-A injection as a treatment. The eyes ipsilateral to the spasm side were used as studied eyes and the contralateral eyes were used as controls. The Ocular Surface Disease Index (OSDI) score, tear break-up time (TBUT), corneal fluorescein staining, and Schirmer I test were measured at baseline, 1 and 3 months after BTX-A injection. Fluorescein clearance test (FCT) was evaluated at baseline and at 1 month after BTX-A injection.

Results: Thirty-one participants ( 6 males and 25 females; mean age $61 \pm 10$ years) were included. The prevalence of DE according to the Asia Dry Eye Society was not significantly different between affected (37.93\%) and non-affected eyes $(27.6 \%) ; \mathrm{P}=0.083$. At baseline, there was no significant difference in TBUT, Schirmer test, basal tear secretion, presence of delayed tear clearance, and presence of reflex tear secretion between affected and nonaffected eyes, while significant difference in Oxford scheme grade was observed $(\mathrm{P}=0.031)$. OSDI score, TBUT, Oxford scheme grade, and Schirmer test at 1 month $(\mathrm{P}=0.817,0.796,0.534,0.556)$, and 3 months $(\mathrm{P}=0.803,0.904,0.936,0.684)$ after BTX-A injection did not significantly change from baseline in affected eyes. FCT results were not significantly different between baseline and at 1-month follow-up in both groups. All findings were corresponding in both naïve and long-term botulinum toxin injection groups. Conclusion: We found no significant effect of BTX-A on signs and symptoms of DE in patients with HFS. Moreover, there was no significant association between HFS and DE. However, we found significant corneal surface damage in the affected eyes, which emphasized importance of ocular surface evaluation and prompt treatment in HFS patients.

Keywords: hemifacial spasm, botulinum toxin, dry eye, fluorescein clearance test, prevalence of dry eye

\section{Introduction}

Hemifacial spasm (HFS) is one of the most common craniofacial movement disorders, which is characterized by irregular, and involuntary clonic and/or tonic movements of muscles innervated by the ipsilateral facial nerve. The etiologies are usually related with aberrant regeneration after facial palsy; and compression of facial nerve at the root exit zone (REZ) by ectatic vessels. HFS is typically unilateral, is more common in females, and occurs in late adulthood. The symptoms of HFS begin in the periorbital musculature and progress to other muscles of facial expression, resulting in functional blindness and reduced quality of life. ${ }^{1-3}$
Correspondence: Supharat Jariyakoso 1873 Rama IV Road, Pathumwan, Bangkok, 10330, Thailand Tel +662-2564l42

Email jsupharat@gmail.com 
Several treatment options for HFS include oral pharmacotherapy, injections of botulinum toxin (BTX) and microvascular decompression. Botulinum toxin injection is currently the mainstay treatment of hemifacial spasm, whereas oral pharmacotherapy or microvascular decompression are beneficial in selected patients.

Botulinum toxin is derived from Clostridium botulinum. The toxin paralyses the injected muscles by irreversible blockage releasing of acetylcholine at the neuromuscular junction. Injection of botulinum toxin type A (BTX-A) has been used worldwide and proved efficacious and safe for treatment of HFS. ${ }^{1,3,4}$ Side effects, including ptosis, tearing, dry eye and diplopia have been reported. . $^{3-7}$

Although the efficacy and side effects of BTX injection for HFS treatment have been well established, the effect of BTX-A on dry eye signs and symptoms is still controversial. $^{5,8-17}$ Furthermore, there are some studies that investigated the association between dry eye and facial dystonia. ${ }^{18-21}$ Only limited studies compared dry eye between affected eye and contralateral non-affected eye in the hemifacial spasm patients. ${ }^{11}$ This study aimed to evaluate the dry eye (DE) in affected and non-affected eyes of HFS patients prior to BTX-A treatment, at 1 month, and 3 months after treated with BTX-A by assessing subjective dry eye symptoms, ocular surface and tear function parameters, and to compare the prevalence of $\mathrm{DE}$ between affected and non-affected eyes in HFS patients.

\section{Patients and Methods}

This prospective observational study was approved by the Faculty of Medicine, Chulalongkorn University's institutional review board and adhered to the tenets of the Declaration of Helsinki. Informed consent was obtained from all patients.

Data were collected from June 2013 to July 2015 at Botulinum Toxin Clinic, King Chulalongkorn Memorial Hospital. Inclusion criteria included participants who were at least 18 years of age with the diagnosis of HFS by experienced neuro-ophthalmologists or neurologists. Exclusion criteria included patients with history of any neurologic abnormalities other than HFS, or with eyelid problems or ocular surface diseases, patients who used any topical medication other than artificial tears within the past 3 months, patients with previous ocular surgery or used contact lens within the past 6 months, patients being treated with neuroleptic or other drugs interfering with eyelid function or neuromuscular transmission, or patients having a contraindication to BTX-A injection. The eyes on the same side of HFS were observed as homolateral eyes, whilst the contralateral non-affected eyes acted as controls. The severity of spasm was graded according to the Jankovic rating scale. $^{22}$

The ophthalmologist, SJ, administered BTX-A injection to all participants soon after ocular surface assessment at baseline. Botulinum toxin type A (Botox; Allergan, Inc., Irvine, CA, USA) was diluted with sterile normal saline to 5 units per $0.1 \mathrm{~mL}$ and applied 2.5 units per site into the preseptal orbicularis oculi muscle of the affected hemiface. Injections were performed in 5 sites ( 2 sites at upper eyelid and 3 sites at lower eyelid) (Figure 1). The other regions of the face were injected with various doses depending on the severity of contraction.

Subjective symptoms of dry eye were evaluated using the Ocular Surface Disease Index (OSDI) questionnaire. After completing the questionnaire, all participants were evaluated tear break-up time (TBUT) and examined the ocular surface by performing fluorescein staining. Consequently, Schirmer I test without anesthesia and fluorescein clearance test (FCT) were performed. All assessments were carried out in both eyes at baseline, 1-month, and 3-month follow-up except for FCT, which were measured at baseline and at 1-month follow-up.

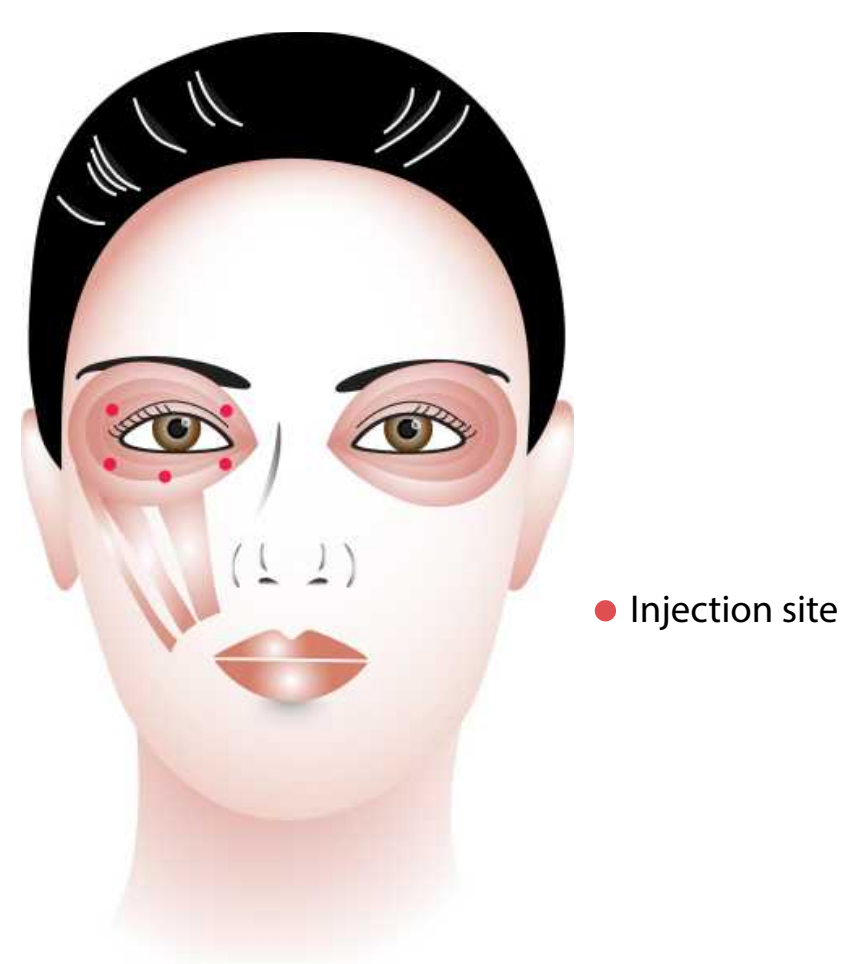

Figure I Dosage and injection sites of botulinum toxin (Image courtesy of Supanut Apinyawasisuk, MD). 
OSDI scores were elicited from the 12-item questionnaire designed to provide a rapid assessment of ocular irritation symptoms consistent with dry eye disease, and the impact on vision-related functioning ${ }^{23}$ was obtained by asking every participant at each visit by a research assistant. To evaluate the stability of tear film, TBUT was measured in seconds (s) and evaluated three times per visit through the use of fluorescence strips and one drop of $0.9 \%$ normal saline. The average TBUT of three measurements was recorded. Also, Oxford scheme grades were obtained under cobalt-blue light after fluorescein staining. The severity of staining was quantified using a chart comprising a series of six panels. In each panel, fluorescein staining is represented by punctate dots. The severity designation from keratoconjunctival staining was rated as stage $0,1,2,3,4$ and $5 .^{24}$ Schirmer's test without anesthetic eye drop was performed by placing Schirmer MARK BLU Tear Test ${ }^{\circledR}$ strips (Optitech Eyecare, Allahabad, India) at inferior fornix of participants for 5 minutes. After 5 minutes, the wetness of the filter paper was measured in millimeter $(\mathrm{mm})$ from the initial fold. FCT determined tear dynamic functions, i.e. basal tear secretion, reflex tear secretion under nasal stimulation and tear clearance at the same time. ${ }^{25}$ One minute after instillation of $0.5 \%$ tetracaine hydrochloride topical anesthetic drops (Alcon Laboratories, Inc., Fort Worth, Texas), 5 microliters of $0.25 \%$ fluorescein sodium with $0.4 \%$ benoxinate hydrochloride solution (OCuSOFT, Inc., Richmond, TX) was applied to the inferior fornix of each eye through an Eppendorf ${ }^{\circledR}$ micropipette (Eppendorf, San Diego, CA) without touching conjunctival tissue with the pipette tip. The participants were asked to blink normally. After a lapse of 10 minutes, a Schirmer strip was placed into inferior fornix at the lateral one-third of the eyelid for 1 minute while the eye was closed. This maneuver was repeated for a total of 3 times every 10 minutes. At the last test at 30 minutes, Schirmer strip was placed after nasal stimulation by using Q-tip put along the lower medial wall inside a nostril. Fluorescence was evaluated by unaided visual inspection under cobalt-blue light.

Dry eye diagnosis was determined following the Asia Dry Eye Society $(\mathrm{ADES})^{26}$ criteria, which included unstable tears film evaluated by TBUT and dry eye symptoms evaluated by relevant dry eye questionnaires, such as OSDI, McMonnies questionnaire, Women's Health Study questionnaire or the dry eye-related QOL score (DEQS). We employed OSDI scores more than 12 and TBUT less than 5 seconds as the ADES criteria for the diagnosis of dry eye. Preservative-free artificial tears were instructed to use at the same frequency in both eyes throughout the study in all participants.

\section{Statistical Analysis}

Demographics and baseline clinical characteristics were reported in mean (standard deviation, SD) and median (first quartile, third quartile). The categorical data was depicted in frequency and percentage.

Paired samples $t$-test, Wilcoxon signed-rank test, and McNemar's test were used to compare data between homolateral and contralateral eyes at baseline. Wilcoxon signed-rank test and McNemar's test were also used to compare between FCT results at baseline and at 1-month follow-up for both homolateral and contralateral eyes. Random intercept linear mixed model analysis was used to analyze OSDI score, TBUT, Oxford scheme grade, and Schirmer's test in between homolateral and contralateral eyes, and results were presented in adjusted means and 95\% confidence intervals (CI).

Subgroup analysis between homolateral and contralateral eyes by naïve and previous BTX-A experience groups on prevalence of DE, and signs of DE including TBUT, Oxford scheme grade, Schirmer's test, basal tear secretion, presence of delayed tear clearance and the presence of reflex tear secretion at baseline was conducted.

For all analyses, an alpha level of 0.05 was adopted for statistical significance, and Stata version 15.1 were used (StataCorp. 2017. Stata Statistical Software: Release 15. College Station, TX: StataCorp LLC).

\section{Results}

A total of 62 eyes; 31 affected eyes with hemifacial spasm (homolateral eye), 31 non-affected eyes (contralateral eye) of 31 participants with unilateral hemifacial spasm were included in the study. Seventeen participants completed a 3-month study period and 14 participants lost to followup at 3-month because of inconvenience and they were out of concern about dry eye status due to progressed HFS disease. There was one participant who did not have FCT result on baseline examination due to incorporation. Moreover, there were no FCT results of four participants at 1-month follow-up because of inconvenience in FCT examination. The baseline clinical parameters and characteristics of participants are reported in Table 1. Eleven out of 31 participants never had any injection of BTX-A as a treatment whereas the rest had at least one injection up to 23 prior injections. 
Table I Baseline Demographics and Clinical Characteristics

\begin{tabular}{|l|c|}
\hline Demographic and Clinical Characteristic & Value (N,\%) \\
\hline Age in years (mean (SD)) & $61(10)$ \\
\hline Sex & $6(19.4 \%)$ \\
Male (\%) & $25(80.6 \%)$ \\
Female (\%) & \\
\hline Side of affected eye & $17(54.8 \%)$ \\
Right (\%) & $14(45.2 \%)$ \\
Left (\%) & \\
\hline Jankovic Rating Scale (JRS) & 8 \\
2 & 18 \\
3 & 5 \\
4 & \\
\hline Number of previous injections of botulinum toxin & \\
type A (n) & 11 \\
0 (naïe) & 10 \\
I-5 & 6 \\
$6-10$ & 4 \\
$>$ I0 & $3(2,3)$ \\
\hline Duration of treatment in years (median (QI,Q3)) & $17.9(4.50,29.54)$ \\
\hline Usage of tear substitutes (\%) & \\
\hline OSDI score (median (QI,Q3)) & \\
\hline
\end{tabular}

Abbreviations: SD, standard deviation; QI, first quartile; Q3, third quartile; OSDI score, Ocular Surface Disease Index score (0-100).

Comparison of prevalence and clinical parameters of dry eye at baseline between homolateral and contralateral eyes are shown in Table 2. There were no statistically significant differences between homolateral and contralateral eyes with respect to prevalence of dry eye defined by ADES criteria, and signs involved with dry eye including TBUT, Schirmer's test, basal tear secretion, delayed tear clearance, and the presence of reflex tear secretion. Only the Oxford scheme grade (median [min, max]) showed statistically significant difference between homolateral eye $(0[0,3]$ and contralateral eye $(0[0,1] ; \mathrm{P}=0.031)$. Moreover, results from subgroup analysis comparing between homolateral and contralateral eyes by naïve and previous BTX-A experience groups showed no statistically significant difference at baseline. In the naïve group, prevalence of dry eye in homolateral eye was $44.44 \%$ and $33.33 \%$ in contralateral eye, which also showed no statistically significant difference $(\mathrm{p}=0.564)$.

Comparisons between baseline and 1-month values of FCT showed no significant difference for each eye (Table 3). According to linear mixed model, pairwise comparisons showed no significant difference in OSDI scores among 3
Table 2 Comparison of Prevalence and Clinical Parameters Between Affected Eyes (Homolateral Eye) and Non-Affected Eyes (Contralateral Eye) at Baseline

\begin{tabular}{|c|c|c|c|}
\hline & $\begin{array}{l}\text { Homolateral } \\
\text { Eye }(n=31)\end{array}$ & $\begin{array}{l}\text { Contralateral } \\
\text { Eye }(n=3 I)\end{array}$ & $\mathbf{P}$ \\
\hline $\begin{array}{l}\text { Prevalence of dry eyes } \\
\text { (\%) }\end{array}$ & $37.93 \%$ & $27.59 \%$ & 0.083 \\
\hline TBUT (s), mean (SD)* & $5.03(0.56)$ & $4.77(0.47)$ & $0.5 \mathrm{II}$ \\
\hline $\begin{array}{l}\text { Oxford scheme grade } \\
(0-5), \text { median (QI, } \\
\text { Q3) }\end{array}$ & $0(0,1)$ & $0(0, I)$ & 0.031 \\
\hline $\begin{array}{l}\text { Schirmer's test }(\mathrm{mm}) \text {, } \\
\text { median }(\mathrm{Q} \mathrm{I}, \mathrm{Q} 3)^{\dagger}\end{array}$ & II $(5,15)$ & $9.5(5,15)$ & 0.424 \\
\hline $\begin{array}{l}\text { Fluorescein clearance } \\
\text { test (FCT) }\end{array}$ & & & \\
\hline $\begin{array}{l}\text { Basal tear secretion } \\
(\mathrm{mm}) \text {, median (QI, } \\
\mathrm{Q} 3)^{\dagger}\end{array}$ & $3.5(2,6)$ & $2.75(I, 7)$ & 0.210 \\
\hline $\begin{array}{l}\text { Delayed tear } \\
\text { clearance (\%) }\end{array}$ & $\begin{array}{l}24 \text { out of } \\
30(80 \%)\end{array}$ & $\begin{array}{c}20 \text { out of } \\
30(66.67 \%)\end{array}$ & 0.157 \\
\hline Reflex tear $(\%)^{\#}$ & $\begin{array}{l}21 \text { out of } \\
30(70 \%)\end{array}$ & $\begin{array}{c}20 \text { out of } \\
30(66.67 \%)\end{array}$ & 0.706 \\
\hline
\end{tabular}

Notes: ${ }^{\dagger} \mathrm{P}$ value by the Wilcoxon signed-rank test; ${ }^{*} \mathrm{P}$ value by the Paired samples $t$-test; ${ }^{\# P}$ value by McNemar's test.

Abbreviations: TBUT, tear break-up time; SD, standard deviation.

time periods (OSDI scores at baseline: 17.61; $\mathrm{CI}=12.51$, 22.71, at 1-month follow-up: 16.24; $\mathrm{CI}=11.10,21.38$, and at 3-month follow-up: 15.85; CI=9.86, 21.83). Adjusted mean of TBUT, Oxford scheme grade, and Schirmer test at baseline, 1and 3-month follow-ups are shown in Figure 2. Pairwise comparison showed no significant difference in TBUT among 3 time periods in both homolateral (TBUT at baseline: 5.04 seconds (s); $\mathrm{CI}=4.02,6.06$, at 1-month follow-up: $5.48 \mathrm{~s}$; $\mathrm{CI}=4.45,6.52$, and at 3-month follow-up: $5.39 \mathrm{~s} ; \mathrm{CI}=4.06$, 6.72) and contralateral group (TBUT at baseline: $4.77 \mathrm{~s}$; $\mathrm{CI}=3.85,5.69$, at 1 -month follow-up: $6.28 \mathrm{~s} ; \mathrm{CI}=5.34,7.21$, and at 3-month follow-up: $5.53 \mathrm{~s} ; \mathrm{CI}=4.31,6.75)$. In addition, pairwise comparisons also showed insignificant difference of Oxford scheme grade among 3 time periods in both homolateral (Oxford scheme grade at baseline: $0.55 ; \mathrm{CI}=0.18,0.92$, at 1-month follow-up: $0.83 ; \mathrm{CI}=0.46,1.21$, and at 3-month follow-up: $0.66 ; \mathrm{CI}=0.18,1.13$ ) and contralateral group (Oxford scheme grade at baseline: $0.36 ; \mathrm{CI}=-0.06,0.78$, at 1-month follow-up: 1.15 ; $\mathrm{CI}=0.74,1.57$, and at 3-month follow-up: $0.40 ; \mathrm{CI}=-0.14,0.94)$ except Oxford scheme grade at 1-month follow-up was significantly different from that at baseline $(\mathrm{P}=0.005)$ and at 3-month follow-up $(\mathrm{P}=0.043)$ in 
Table 3 Comparison of Fluorescein Clearance Test (FCT) Between FCT at Baseline and FCT at I Month After BTX-A Injection in Homolateral and Contralateral Eye

\begin{tabular}{|c|c|c|c|}
\hline & Baseline $(n=30)$ & $\begin{array}{l}\text { I-Month Follow-Up } \\
(n=27)\end{array}$ & $\mathbf{P}$ \\
\hline \multicolumn{4}{|l|}{ Homolateral eye } \\
\hline Basal tear secretion $(\mathrm{mm})$, median $(\mathrm{Q} \mathrm{I}, \mathrm{Q} 3)^{\dagger}$ & $3.5(2,6)$ & $5(1,9)$ & 0.424 \\
\hline Delayed tear clearance $(\%)^{\#}$ & 20 out of $26(76.92 \%)$ & 24 out of $26(92.31 \%)$ & 0.103 \\
\hline Reflex tear $(\%)^{\#}$ & 17 out of $26(65.38 \%)$ & II out of $26(42.31 \%)$ & 0.083 \\
\hline \multicolumn{4}{|l|}{ Contralateral eye } \\
\hline Basal tear secretion $(\mathrm{mm})$, median $(\mathrm{Q} \mathrm{I}, \mathrm{Q} 3)^{\dagger}$ & $2.75(1,7)$ & $3(1,6)$ & 0.308 \\
\hline Delayed tear clearance $(\%)^{\#}$ & 16 out of $26(61.54 \%)$ & 21 out of $26(80.77 \%)$ & 0.059 \\
\hline Reflex tear $(\%)^{\#}$ & 16 out of $26(61.54 \%)$ & 17 out of $26(65.38 \%)$ & 0.706 \\
\hline
\end{tabular}

Notes: ${ }^{\dagger} \mathrm{P}$ value by the Wilcoxon signed-rank test; ${ }^{\#} \mathrm{P}$ value by McNemar's test.

contralateral eye. Pairwise comparisons of Schirmer's test among 3 time periods also showed no significance in both homolateral (Schirmer test at baseline: $11.57 \mathrm{~mm}$; $\mathrm{CI}=8.38$, 14.75, at 1 -month follow-up: $9.43 \mathrm{~mm} ; \mathrm{CI}=6.18,12.67$, and at 3-month follow-up: $13.73 \mathrm{~mm} ; \mathrm{CI}=9.34,18.12)$ and contralateral eyes (Schirmer test at baseline: $12.17 \mathrm{~mm} ; \mathrm{CI}=8.57$, 15.77, at 1-month follow-up: $9.54 \mathrm{~mm} ; \mathrm{CI}=5.90,13.18$, and at 3-month follow-up: $11.08 \mathrm{~mm} ; \mathrm{CI}=6.51,15.65)$.
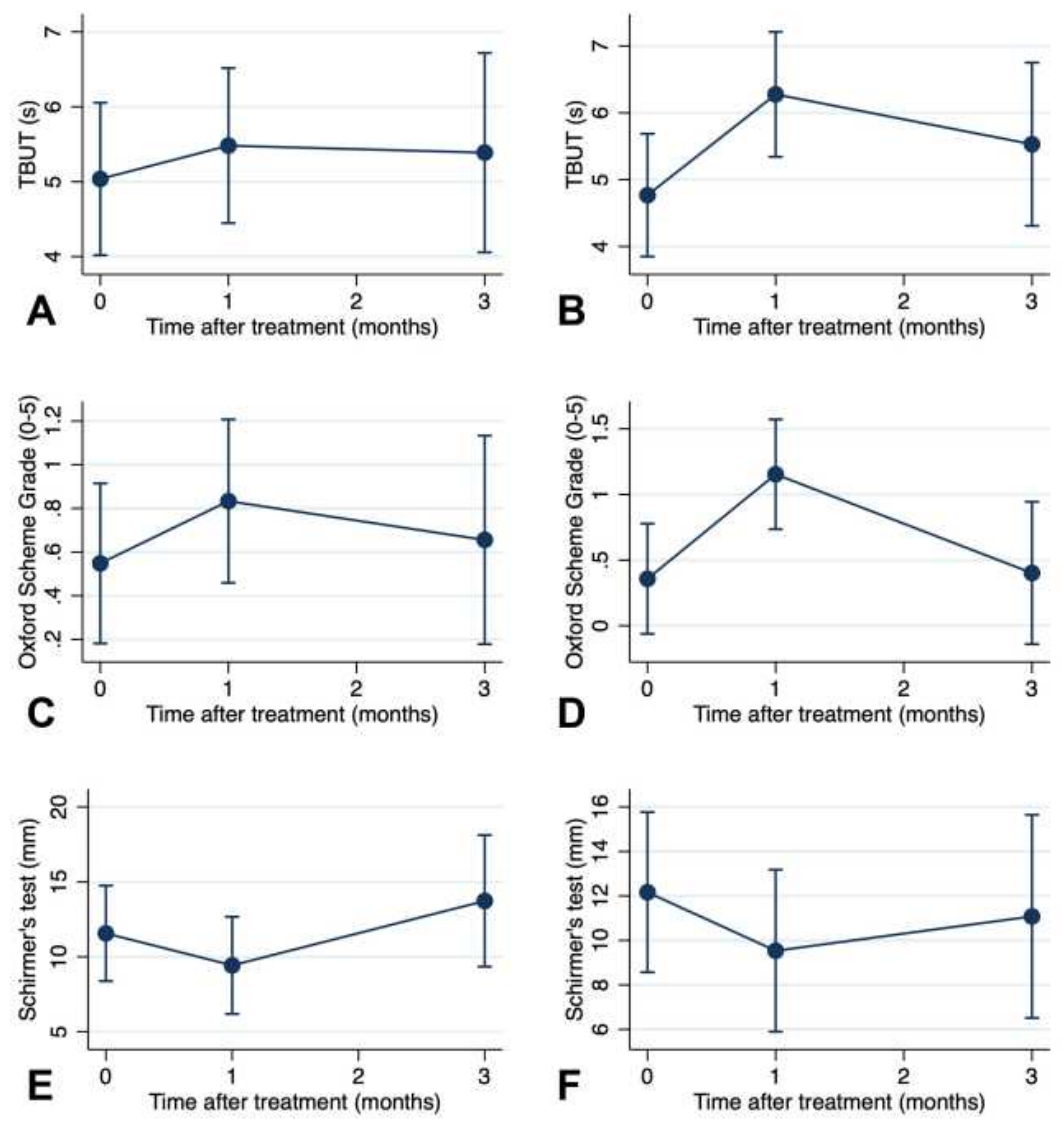

Figure 2 Dry eye parameters at baseline, I-month, and 3-month after injection of botulinum toxin type A in homolateral and contralateral eyes: (A) tear break-up time of homolateral eyes; (B) tear break-up time of contralateral eyes; (C) Oxford scheme grade of homolateral eyes; (D) Oxford scheme grade of contralateral eyes; (E) Schirmer's test of homolateral eyes; (F) Schirmer's test of contralateral eyes. 


\section{Discussion}

To the best of our knowledge, our study is the first to compare the prevalence of DE between affected and nonaffected eyes in hemifacial spasm patients prior to BTX-A treatment. Our observed prevalence of DE defined by ADES criteria in homolateral eye (37.93\%) was not statistically significantly higher than that in contralateral eye (27.6\%), resulting in approximately ten-percent difference that could be deemed as a clinical importance. To remove the effect of long-term botulinum toxin use, we investigated DE in the naïve group (11 patients, 35.5\%) which comprise of patients who had botulinum toxin injection for the first time and found no statistical difference in prevalence of dry eye between homolateral eyes and contralateral eye ( $44.44 \%$ and $33.33 \%$, respectively). The observed prevalence of DE among naïve group is similar to that of DE among HFS patients reported by Raj et al ${ }^{20}(47.06 \%)$ and Pellegrini et $\mathrm{al}^{11}(42.3 \%)$ using TFOS DEWS II criteria.

At baseline, TBUT and Schirmer test in homolateral eyes were not significantly different from contralateral eyes. Moreover, our study showed insignificant differences of tear dynamic by using FCT including basal tear secretion, presence of delayed tear clearance, and presence of reflex tear secretion between two eyes. However, the observed higher baseline Oxford scheme grade indicating poorer health of corneal epithelial cells ${ }^{27}$ in homolateral eye might be caused by HFS. We conceptualized that spasm of affected eyes with forceful eyelid closure and increase of blink rate contributed to microtrauma of ocular surface damage. This was supported by Yamaguchi and Shiraishi who found a significant correlation between high eyelid pressure and ocular surface staining. ${ }^{28}$

Our findings showed no significant association between HFS and DE, which was supported by Price and O'Day reporting no significant difference in tear secretion between HFS and control groups. ${ }^{21}$ We found no statistically significant difference in TBUT, Schirmer test and FCT results between affected and non-affected eyes at baseline in both naïve and long-term botulinum toxin injection groups. These were different from Pellegrini et al, where TBUT, Schirmer's test and corneal sensitivity in homolateral eyes were significantly lower than contralateral eyes. However, Pellegrini et al investigated among subjects with a history of multiple botulinum toxin injections. One might suspect that ocular surface impairment might relate to long-term effect of BTX-injection, hemifacial spasm itself, or combined factors. ${ }^{11}$ Our findings on the HFS patients naïve to botulinum toxin add different aspect to the current knowledge and may clarify the suspected insignificant association between DE and HFS.

The impact of BTX-A on dry eye was still inconclusive and controversial. Controlling for potential confounding factors through comparisons with non-affected eyes, our study showed that OSDI score was not significantly changed over 3-month follow-up and that signs of tear dynamic including FCT, TBUT and Schirmer's test were not significantly changed in both eyes. Our results indicated that BTX-A injection did not significantly cause tear film change. Few studies investigating only affected eyes of HFS patients showed that the injection of BTX-A caused decreased tear film stability and decreased Schirmer test, especially in patients received lateral injection sites. ${ }^{5,7,14}$ Mitigating effects including an increase in tear meniscus height, reduction of mean blink output, an increase in lipid tear thickness, and an increased TBUT following BTX-A injection were also observed from the other studies with similar design..$^{5,7,12}$ To the best of our knowledge, only a study by Pellegrini et al had non-affected eye as a comparison and found a significant lower of TBUT at 1 month after injection of BTX-A in affected eye, but no significant change in non-affected eye among HFS patients who received long-term BTX-A injections. ${ }^{11}$ Our insignificant observations on tear dynamic with a unique design including naïve and long-term BTX-A injection of HFS patients added to the pool of conflicting evidence of the BTX-A effect on tear dynamic. ${ }^{5,7,12,14}$ We suspect that BTX-A may play a role in tear dynamic and present its effects through changes in tear secretion from lacrimal gland, lipid layer thickness, amount of tear on ocular surface, or tear film. The discernable BTX-A effects would depend on BTX-A dosage and concentration, injection technique, injection sites, and size of diffusion area of BTX-A to surrounding tissue. The future investigations should consider these issues to further identify the specific effects of BTX-A on DE.

In addition, our study strength includes the control of other confounding factors through having non-affected eyes as a control. Other potential confounding factors affecting DE such as age, gender, race, smoking, environmental stimuli or computer use $\mathrm{e}^{29}$ were controlled for in our current study since non-affected eyes were used as control. 
There were some limitations in this study. Nonpreservative artificial tears were used by some patients; however, the effects may be minimal even with different compositions in various types of artificial tears. The frequency of applying tear substitutes was not restricted to a specific time per day due to personal preference; however, each participant used the same preferred frequency throughout the study and on both homolateral and contralateral eyes equally. The similarity of change in dry eye parameters and trends in both eyes (Figure 2) might be partially result from various frequency in tear substitute use and equal application on both eyes. The other potential limitations consisted of small sample size and high rate of loss-to-follow-up. The majority of missing data of the loss-to-follow-up patients was at 3 months (45.2\%), where, at the point in time, a comparison of dry eye between both eyes was deemed to be less important due to the wean-off effect of BTX-A. In contrast, BTX-A effects at 1 month was concerning and considered to be the peak effect of the medication. Nevertheless, future study with a larger number of subjects might be beneficial to investigate the association between dry eye and hemifacial spasm.

\section{Conclusion}

To conclude, we presumed that there was insignificant association between HFS and dry eye, which was corresponding in both naïve and long-term botulinum toxin injection groups. The botulinum toxin injection did not significantly affect signs of tear film change over time in HFS patients. However, we found significant corneal surface damage in the affected eye of HFS patients. This damage raised the importance of an ocular surface evaluation and a prompt treatment in patients with hemifacial spasm.

\section{Acknowledgments}

This study was supported by Ratchadapiseksompotch Fund, Faculty of Medicine, Chulalongkorn University (Grant no RA 56/028) and Grants for Development of New Faculty Staff, Ratchadaphiseksomphot Endowment Fund, Chulalongkorn University.

\section{Disclosure}

The authors report no conflicts of interest in this work.

\section{References}

1. Tan NC, Chan LL, Tan EK. Hemifacial spasm and involuntary facial movements. QJM. 2002;95(8):493-500. doi:10.1093/qjmed/95.8.493
2. Kenney C, Jankovic J. Botulinum toxin in the treatment of blepharospasm and hemifacial spasm. J Neural Transm (Vienna). 2008;115 (4):585-591. doi:10.1007/s00702-007-0768-7

3. Chaudhry N, Srivastava A, Joshi L. Hemifacial spasm: the past, present and future. $J$ Neurol Sci. 2015;356(1-2):27-31. doi:10.1016/j. jns.2015.06.032

4. Sorgun MH, Yilmaz R, Akin YA, Mercan FN, Akbostanci MC Botulinum toxin injections for the treatment of hemifacial spasm over 16 years. J Clin Neurosci. 2015;22(8):1319-1325. doi:10.1016/ j.jocn.2015.02.032

5. Ho RW, Fang PC, Chao TL, Chien CC, Kuo MT. Increase lipid tear thickness after botulinum neurotoxin A injection in patients with blepharospasm and hemifacial spasm. Sci Rep. 2018;8(1):8367. doi:10.1038/s41598-018-26750-5

6. Bentivoglio AR, Fasano A, Ialongo T, Soleti F, Lo Fermo S, Albanese A. Outcome predictors, efficacy and safety of botox and dysport in the long-term treatment of hemifacial spasm. Eur J Neurol. 2009;16(3):392-398. doi:10.1111/j.1468-1331.2008.02507.x

7. Ho RW, Fang PC, Chang CH, Liu YP, Kuo MT. A review of periocular botulinum neurotoxin on the tear film homeostasis and the ocular surface change. Toxins (Basel). 2019;11(2):66. doi:10.3390/toxins11020066

8. Alsuhaibani AH, Eid SA. Botulinum toxin injection and tear production. Curr Opin Ophthalmol. 2018;29(5):428-433. doi:10.1097/ICU.00000 00000000506

9. Gunes A, Demirci S, Koyuncuoglu HR, Tok L, Tok O. Corneal and tear film changes after botulinum toxin-A in blepharospasm or hemifacial spasm. Cornea. 2015;34(8):906-910. doi:10.1097/ICO.0000000000000482

10. Ziak P, Halicka J, Kapitanova K, Mojzis P. Effect of botulinum toxin A application in neuro-ophtalmologic indications on schirmers test and tears osmolarity. Cesk Slov Oftalmol. 2019;75(2):74-77. doi:10.31348/ 2019/2/3

11. Pellegrini M, Schiavi C, Taroni L, et al. Ocular surface status in patients with hemifacial spasm under long-lasting treatment with botulinum A toxin: a Comparative Fellow Eye Study. Indian J Ophthalmol. 2019;67(9):1405-1409. doi:10.4103/ijo.IJO 4119

12. Park DI, Shin HM, Lee SY, Lew H. Tear production and drainage after botulinum toxin A injection in patients with essential blepharospasm. Acta Ophthalmol. 2013;91(2):e108-112. doi:10.1111/aos.12002

13. Mali YP, Schultze RL, Wladis EJ. Evaluating the effects of botulinum toxin A on tear metrics in patients with hemifacial spasm. Orbit. 2017;36(5):307-310. doi:10.1080/01676830.2017.1337164

14. Horwath-Winter J, Bergloeff J, Floegel I, Haller-Schober EM, Schmut O. Botulinum toxin A treatment in patients suffering from blepharospasm and dry eye. Br J Ophthalmol. 2003;87(1):54-56. doi:10.1136/bjo.87.1.54

15. Lu R, Huang R, Li K, et al. The influence of benign essential blepharospasm on dry eye disease and ocular inflammation. Am JOphthalmol. 2014;157(3):591-597.e591-592. doi:10.1016/j.ajo.2013.11.014

16. Kocabeyoglu S, Sekeroglu HT, Mocan MC, Muz E, Irkec M, Sanac AS. Ocular surface alterations in blepharospasm patients treated with botulinum toxin A injection. Eur J Ophthalmol. 2014;24 (6):830-834. doi:10.5301/ejo.5000482

17. Spiera H, Asbell PA, Simpson DM. Botulinum toxin increases tearing in patients with Sjogren's syndrome: a preliminary report. J Rheumatol. 1997;24(9):1842-1843.

18. Tsubota K, Fujihara T, Kaido M, Mori A, Mimura M, Kato M. Dry eye and Meige's syndrome. Br J Ophthalmol. 1997;81(6):439-442. doi:10.1136/bjo.81.6.439

19. Gosal JS, Das KK, Khatri D, Attri G, Jaiswal AK. Contralateral dry eye in hemifacial spasm: a new clinical sign. Asian J Neurosurg. 2019;14(3):996-998. doi:10.4103/ajns.AJNS_48_19

20. Raj A, Arya SK, Deswal J, Bamotra RK. Five-year retrospective review of cases with benign essential blepharospasm and hemifacial spasm presenting in a tertiary eye care center in North India. Semin Ophthalmol. 2017;32(3):371-376. doi:10.3109/08820538.2015. 1096401 
21. Price J, O’Day J. A comparative study of tear secretion in blepharospasm and hemifacial spasm patients treated with botulinum toxin. J Clin Neuroophthalmol. 1993;13(1):67-71.

22. Jankovic J, Kenney C, Grafe S, Goertelmeyer R, Comes G. Relationship between various clinical outcome assessments in patients with blepharospasm. Mov Disord. 2009;24(3):407-413. doi: $10.1002 / \mathrm{mds} .22368$

23. Miller KL, Walt JG, Mink DR, et al. Minimal clinically important difference for the ocular surface disease index. Arch Ophthalmol. 2010;128(1):94-101. doi:10.1001/archophthalmol.2009.356

24. Bron AJ, Evans VE, Smith JA. Grading of corneal and conjunctival staining in the context of other dry eye tests. Cornea. 2003;22 (7):640-650. doi:10.1097/00003226-200310000-00008

25. Maskin SL. Effect of ocular surface reconstruction by using amniotic membrane transplant for symptomatic conjunctivochalasis on fluorescein clearance test results. Cornea. 2008;27(6):644-649. doi:10.1097/01.ico.0000611376.96794.01
26. Tsubota K, Yokoi N, Shimazaki J, et al. New perspectives on dry eye definition and diagnosis: a consensus report by the Asia dry eye society. Ocul Surf. 2017;15(1):65-76. doi:10.1016/j.jtos.2016.09.003

27. Wolffsohn JS, Arita R, Chalmers R, et al. TFOS DEWS II diagnostic methodology report. Ocul Surf. 2017;15(3):539-574.

28. Yamaguchi M, Shiraishi A. Relationship between eyelid pressure and ocular surface disorders in patients with healthy and dry eyes. Invest Ophthalmol Vis Sci. 2018;59(14):Des56-des63. doi:10.1167/iovs.1723586

29. Sweeney DF, Millar TJ, Raju SR. Tear film stability: a review. Exp Eye Res. 2013;117:28-38. doi:10.1016/j.exer.2013.08.010
Clinical Ophthalmology

\section{Publish your work in this journal}

Clinical Ophthalmology is an international, peer-reviewed journal covering all subspecialties within ophthalmology. Key topics include: Optometry; Visual science; Pharmacology and drug therapy in eye diseases; Basic Sciences; Primary and Secondary eye care; Patient Safety and Quality of Care Improvements. This journal is indexed on PubMed

\section{Dovepress}

Central and CAS, and is the official journal of The Society of Clinical Ophthalmology (SCO). The manuscript management system is completely online and includes a very quick and fair peer-review system, which is all easy to use. Visit http://www.dovepress.com/ testimonials.php to read real quotes from published authors. 